\title{
REGULAR HOMOTOPIC DEFORMATION OF COMPACT SURFACE WITH BOUNDARY AND MAPPING CLASS GROUP
}

\author{
SUSUMU HIROSE AND AKIRA YASUHARA
}

\begin{abstract}
A necessary and sufficient algebraic condition for a diffeomorphism over a surface embedded in $S^{3}$ to be induced by a regular homotopic deformation is discussed, and a formula for the number of signed pass moves needed for this regular homotopy is given.
\end{abstract}

\section{INTRODUCTION}

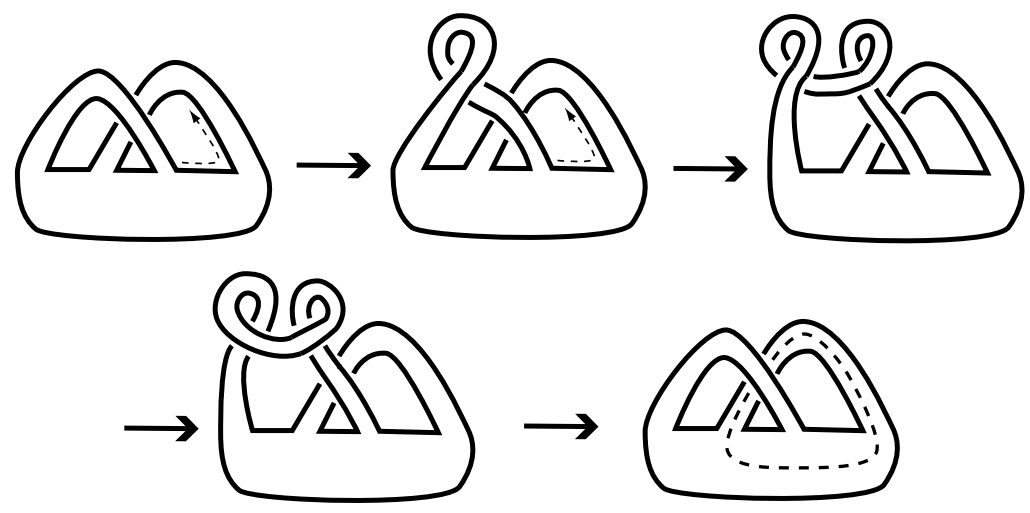

Figure 1. An example of regular homotopic deformation of a torus with one boundary. The result is a double Dehn-twist about the dashed circle in the last surface.

Two embeddings $f_{1}$ and $f_{2}$ of a surface $F$ into $S^{3}$ are called regular homotopic $f_{1} \sim f_{2}$, if there is a homotopy $h_{t}(0 \leq t \leq 1)$ such that $h_{0}=f_{1}, h_{1}=f_{2}$, and $h_{t}$ is an immersion of $F$ into $S^{3}$ for each $t \in[0,1]$. Figure 1 is an example of regular homotopy. This regular homotopy induces a double Dehn-twist about the dashed circle drawn in the last surface. That is to say, some regular homotopic deformation induces an isotopically non-trivial diffeomorphism on the surface. In $\S 2$, we gives a necessary and sufficient algebraic condition for a diffeomorphism over the surface to

2000 Mathematics Subject Classification. 57M60, 57N10.

This research was supported by Grant-in-Aid for Scientific Research (C) (No. 20540083 and No. 20540065), Japan Society for the Promotion of Science. 
be induced by a regular homotopic deformation. In this section, we observe that, in order to perform the above regular homotopy, pass-moves are enough. In $\S 3$, we give a formula which gives a number of pass-moves we need.

\section{An ALGEBRAIC CHARACTERIZATION OF A MAP INDUCED BY A REGUlaR HOMOTOPIC DEFORMATION}

Let $F$ be a compact oriented surface with boundary, and let $f$ be a smooth embedding of $F$ into $S^{3}$. Let $\mathcal{M}_{F}$ be the mapping class group of $F$, namely $\mathcal{M}_{F}=$ $\pi_{0}\left(\left\{\phi \in \operatorname{Diff}_{+}(F) ;\left.\phi\right|_{\partial F}=i d_{\partial F}\right\}\right)$. In this paper, we mainly investigate on the subgroup of $\mathcal{M}_{F}$ defined by

$$
\mathcal{E}_{f}=\left\{\phi \in \mathcal{M}_{F} ; f \circ \phi \text { is regular homotopic to } f\right\} .
$$

We remark here that, in the above regular homotopy, the boundary of $F$ can be moved. We define a quadratic form $q_{f}: H_{1}\left(F ; \mathbb{Z}_{2}\right) \rightarrow \mathbb{Z}_{2}$ associated the embed$\operatorname{ding} f$ by $q_{f}(x)=l k\left(f_{*}(x), f_{*}(x)^{+}\right) \bmod 2$, where $f_{*}(x)^{+}$is $f_{*}(x)$ pushed into the positive normal direction of $f(F)$. Let $\mathcal{O}_{q_{f}}$ be the subgroup of $\mathcal{M}_{F}$ defined by $\mathcal{O}_{q_{f}}=\left\{\phi \in \mathcal{M}_{F} ; q_{f}\left(\phi_{*}(x)\right)=q_{f}(x)\right.$ for all $\left.x \in H_{1}\left(F ; \mathbb{Z}_{2}\right)\right\}$. Then we have the following theorem.

Theorem 1. $\mathcal{E}_{f}=\mathcal{O}_{q_{f}}$.

Remark 2. Nowik [2] proved the same type theorem for closed surface.

In order to proof this theorem, we will use the following fact. Although this fact is already proven by Pinkall [3], we will prove this by an elementary method.

Lemma 3. Embeddings $f$ and $f^{\prime}$ of $F$ into $S^{3}$ are regular homotopic if and only if $q_{f}=q_{f^{\prime}}$.

Proof. At first, we show that if $f$ and $f^{\prime}$ are regular homotopic then $q_{f}=q_{f^{\prime}}$. For any $x \in H_{1}\left(F ; \mathbb{Z}_{2}\right)$, we take a simple closed curve $\xi$ on $F$ which represents $x$ and let $N(\xi)$ be a regular neighborhood of $\xi$ in $F$. Since $f$ and $f^{\prime}$ are regular homotopic, $\left.f\right|_{N(\xi)}$ and $\left.f^{\prime}\right|_{N(\xi)}$ are regular homotopic. Let $f(\xi)^{+}\left(\right.$resp. $\left.f^{\prime}(\xi)^{+}\right)$be a simple closed curve which is $f(\xi)$ (resp. $f^{\prime}(\xi)$ ) pushed into the positive normal direction 
of $f(N(\xi))$ (resp. $\left.f^{\prime}(N(\xi))\right)$, then $l k\left(f(\xi), f(\xi)^{+}\right)=l k\left(f^{\prime}(\xi), f^{\prime}(\xi)^{+}\right) \bmod 2$ by [1]. Therefore, $q_{f}(x)=q_{f^{\prime}}(x)$.

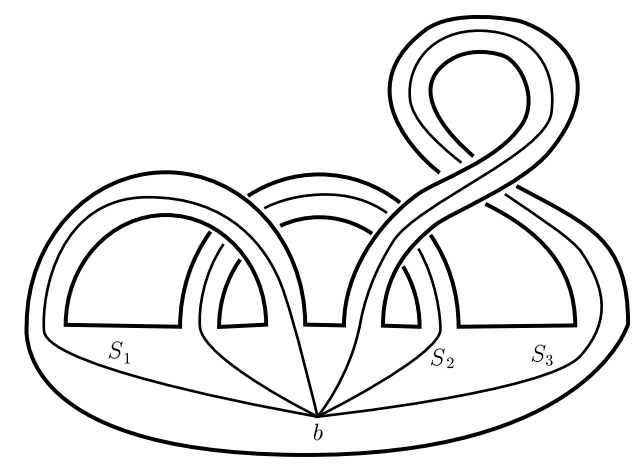

Figure 2. An example of spine

Next, we prove that if $q_{f}=q_{f^{\prime}}$ then $f$ and $f^{\prime}$ are regular homotopic. Let $g$ be the genus of $F$ and $k$ be the number of connected components of $\partial F$. Then, as drawn in Figure 2, we take a bouquet $S$ in $F$ which is constructed from $2 g+k-1$ simple closed curves $S_{1}, \ldots, S_{2 g+k-1}$ by gluing in one point $b$, such that $F \backslash S$ is $k$ annuli. This $S$ is called a spine of $F$. Let $N\left(S_{i}\right)$ be a thin regular neighborhood of $S_{i}$ in $F$ and $N(S)$ be the union of $N\left(S_{1}\right), \ldots, N\left(S_{2 g+k-1}\right)$, then $N(S)$ is a regular neighborhood of $S$ in $F$. Let $r_{S}$ be an embedding from $F$ to itself such that $r_{S}(F)=N(S),\left.r_{S}\right|_{S}=i d$, and $r_{S}$ is isotopic to the identity. By a regular homotopy, we deform $f$ and $f^{\prime}$ such that $f(b)=f^{\prime}(b)=b$. Since $q_{f}=q_{f^{\prime}}, q_{f}\left(\left[S_{i}\right]\right)=q_{f^{\prime}}\left(\left[S_{i}\right]\right)$ for each $i$, in other word, $l k\left(f\left(S_{i}\right), f\left(S_{i}\right)^{+}\right)=l k\left(f^{\prime}\left(S_{i}\right), f^{\prime}\left(S_{i}\right)^{+}\right) \bmod 2$ for each $i$. By this condition on linking numbers, we can construct a regular homotopy between $\left.f\right|_{N\left(S_{i}\right)}$ and $\left.f^{\prime}\right|_{N\left(S_{i}\right)}$ which is a composition of pass moves (see Figure 3) and isotopies as follows. Since pass moves are crossing changes for the knot $f\left(S_{i}\right)$ in $S^{3}$, we can change $f\left(S_{i}\right)$ into $f^{\prime}\left(S_{i}\right)$ by some pass moves and isotopies. Let $f_{1}$ be the embedding of $N\left(S_{i}\right)$ regular homotopic to $\left.f\right|_{N\left(S_{i}\right)}$ which is obtained as a result of above pass moves and isotopies. Then, by the previous condition on linking numbers, the difference between the number of twists of $f_{1}\left(N\left(S_{i}\right)\right)$ and that of $f^{\prime}\left(N\left(S_{i}\right)\right)$ is an even integer. Since this difference is dissolved by pass moves (see Figure 4), we can construct regular homotopy between $f_{1}$ and $\left.f^{\prime}\right|_{N\left(S_{i}\right)}$, and as a result, between $\left.f\right|_{N\left(S_{i}\right)}$ and $\left.f^{\prime}\right|_{N\left(S_{i}\right)}$. Since $N(S)$ is a union of $N\left(S_{i}\right)$ 's, we can construct a regular homotopy 
between $\left.f\right|_{N(S)}$ and $\left.f^{\prime}\right|_{N(S)}$. From the above construction, we can obtain a sequence of regular homotopies, $f \sim f \circ r_{S}=\left(\left.f\right|_{N(S)}\right) \circ r_{S} \sim\left(\left.f^{\prime}\right|_{N(S)}\right) \circ r_{S}=f^{\prime} \circ r_{S} \sim f^{\prime}$. Therefore, $f$ is regular homotopic to $f^{\prime}$.

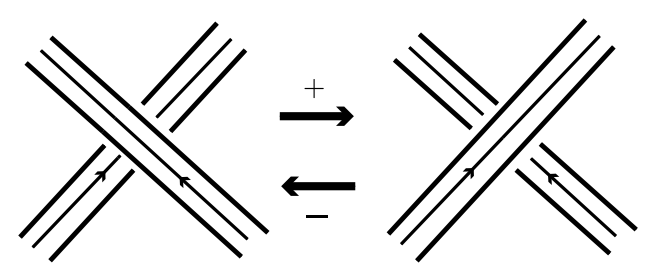

Figure 3. Left to right is + pass move and right to left is - pass move

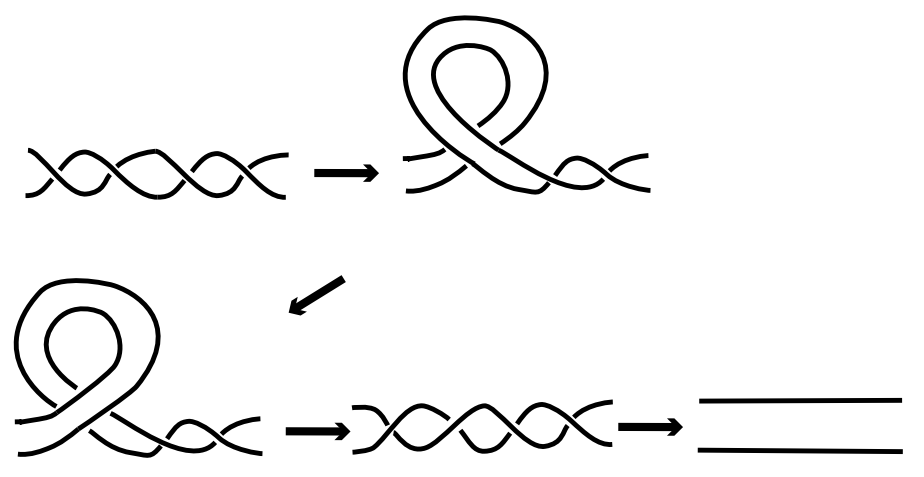

Figure 4. A pass move is used in the second move

Now, we prove Theorem 1 .

Proof of Theorem 1. We remark that $q_{f} \circ \phi_{*}=q_{f \circ \phi}$. If $\phi \in \mathcal{O}_{q_{f}}$ then $q_{f \circ \phi}=q_{f} \circ \phi_{*}=$ $q_{f}$. By Lemma 3, $f \circ \phi$ is regular homotopic to $f$, in other word, $\phi \in \mathcal{E}_{f}$. On the other hand, if $\phi \in \mathcal{E}_{f}$, then $f \circ \phi$ is regular homotopic to $f$. By Lemma 3, $q_{f \circ \phi}=q_{f}$, that is to say, $q_{f} \circ \phi_{*}=q_{f}$.

Furthermore, we see that $\mathcal{E}_{f}$ completely determines the regular homotopy class of $f$.

Proposition 4. $f$ is regular homotopic to $f^{\prime}$ if and only if $\mathcal{E}_{f}=\mathcal{E}_{f^{\prime}}$

Proof. We assume that $f$ is regular homotopic to $f^{\prime}$. If $\phi \in \mathcal{E}_{f}$, then $f \circ \phi \sim f$. Therefore, $f^{\prime} \circ \phi \sim f \circ \phi \sim f \sim f^{\prime}$, hence, $\phi \in \mathcal{E}_{f}^{\prime}$. Similarly, we can see that if $\phi \in \mathcal{E}_{f}^{\prime}$ then $\phi \in \mathcal{E}_{f}$. 
On the other hand, we assume that $f \nsim f^{\prime}$. Then, by Lemma 3, $q_{f} \neq q_{f^{\prime}}$. Therefore, there is an element $\alpha$ of $H_{1}\left(F ; \mathbb{Z}_{2}\right)$ such that $q_{f}(\alpha) \neq q_{f^{\prime}}(\alpha)$, say $q_{f}(\alpha)=$ $1, q_{f^{\prime}}(\alpha)=0$. Let $a$ be a simple closed curve on $F$ which represents $\alpha$, then $T_{a} \in \mathcal{O}_{q_{f}}$ but $T_{a} \notin \mathcal{O}_{q_{f^{\prime}}}$. By Theorem 1, $T_{a} \in \mathcal{E}_{f}$ but $T_{a} \notin \mathcal{E}_{f^{\prime}}$. Therefore, $\mathcal{E}_{f} \neq \mathcal{E}_{f^{\prime}}$.

\section{A FORMUlA OF A SIGNED NUMBER OF PASS MOVES}

We remark that, in the proof of Lemma 3, we use only pass moves and isotopies as a regular homotopy between $f$ and $f^{\prime}$. We call a regular homotopy which is a composition of pass moves and isotopies pass homotopy. In the following, we investigate on some formula which gives a number of "signed" pass moves.

As in the proof of Lemma 3, we choose a spine $S=S_{1} \vee \cdots \vee S_{2 g+k-1}$ of $F$, and orient the simple closed curves $S_{i}$. A pass move which changes a negative crossing into a positive crossing is called a + pass move, and a pass move which changes a positive crossing into a negative crossing is called a - pass move (see Figure 3). We choose a basis $\left\{\alpha_{i} ; 1 \leq i \leq 2 g+k-1\right\}$ of $H_{1}(F ; \mathbb{Z})$ by $\alpha_{i}=\left[S_{i}\right]$, and determine a quadratic form $\tilde{q}_{f}: H_{1}(F ; \mathbb{Z}) \rightarrow \mathbb{Z}$ by $\tilde{q}_{f}(x)=l k\left(f_{*}(x), f_{*}(x)^{+}\right)$.

Theorem 5. Let $\phi$ be an element of $\mathcal{E}_{f}$. For any pass homotopy from $f$ to $f \circ \phi$, ( the number of + pass moves $)-($ the number of - pass moves $)$ $=\frac{1}{2}\left\{\tilde{q}_{f}\left((\phi)_{*}\left(\alpha_{1}+\alpha_{2}+\cdots+\alpha_{2 g+k-1}\right)\right)-\tilde{q}_{f}\left(\alpha_{1}+\alpha_{2}+\cdots+\alpha_{2 g+k-1}\right)\right\}$.

Proof. By Theorem 1, $q_{f}\left(\phi_{*}(x)\right)=q_{f}(x)$ for all $x \in H_{1}\left(F ; \mathbb{Z}_{2}\right)$, hence, $\tilde{q}_{f}\left((\phi)_{*}\left(\alpha_{1}+\right.\right.$ $\left.\left.\alpha_{2}+\cdots+\alpha_{2 g+k-1}\right)\right)-\tilde{q}_{f}\left(\alpha_{1}+\alpha_{2}+\cdots+\alpha_{2 g+k-1}\right)$ is an even integer. Since a linking form is bilinear,

$$
\begin{aligned}
& \frac{1}{2}\left\{\tilde{q}_{f}\left((\phi)_{*}\left(\alpha_{1}+\alpha_{2}+\cdots+\alpha_{2 g+k-1}\right)\right)-\tilde{q}_{f}\left(\alpha_{1}+\alpha_{2}+\cdots+\alpha_{2 g+k-1}\right)\right\} \\
& \quad=\frac{1}{2}\left\{\sum_{i, j} l k\left((f \circ \phi)_{*}\left(\alpha_{i}\right),\left((f \circ \phi)_{*}\left(\alpha_{j}\right)\right)^{+}\right)-l k\left(f_{*}\left(\alpha_{i}\right),\left(f_{*}\left(\alpha_{j}\right)\right)^{+}\right)\right\} .
\end{aligned}
$$

Here, we set

$$
\Delta_{i, j}=l k\left((f \circ \phi)_{*}\left(\alpha_{i}\right),\left((f \circ \phi)_{*}\left(\alpha_{j}\right)\right)^{+}\right)-l k\left(f_{*}\left(\alpha_{i}\right),\left(f_{*}\left(\alpha_{j}\right)\right)^{+}\right) .
$$

We assume that $f \circ \phi$ is obtained from $f$ are regular homotpic by one pass move. We determine the value of $\Delta_{i, j}$ according to the cases on the pass move (see Figure 


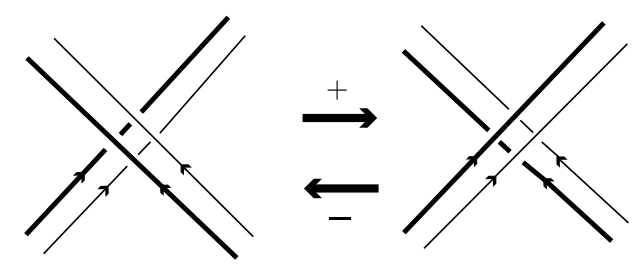

Figure 5. Thick line is a curve in the spine, and thin line is a curve in the spine pushed off to the positive normal direction

5). If the pass move is the + pass move (resp. - pass move) among $S_{i_{0}}$ and itself, then

$$
\Delta_{i, j}= \begin{cases}+2 \quad(\text { resp. }-2) & i=j=i_{0} \\ 0 \quad(\text { resp. }) & \text { otherwise. }\end{cases}
$$

If the pass move is the + pass move (resp. - pass move) among $S_{i_{0}}$ and $S_{j_{0}}\left(i_{0} \neq j_{0}\right)$, then

$$
\Delta_{i, j}=\left\{\begin{array}{lll}
+1 & (\text { resp. }-1) & (i, j)=\left(i_{0}, j_{0}\right) \\
+1 & (\text { resp. }-1) & (i, j)=\left(j_{0}, i_{0}\right) \\
0 \quad(\text { resp.0 }) & \text { otherwise. }
\end{array}\right.
$$

In each cases, for the + pass move, $\sum_{i, j} \Delta_{i, j}=+2$, for the - pass move, $\sum_{i, j} \Delta_{i, j}=$ -2 .

In general, there are several pass moves in a pass homotopy from $f$ to $f \circ \phi$. By applying the same argument as above for each pass move, we show the equation we need.

Remark 6. We remark that the number of signed pass moves of Theorem 5 depends on a choice of an oriented spine of $F$. We choose an oriented spine $S$ in $F$. Then, by Theorem 5 ,

$$
\mathcal{P}_{S}(\phi)=(\text { the number of }+ \text { pass moves })-(\text { the number of }- \text { pass moves })
$$

is well-defined as a function of $\phi \in \mathcal{E}_{f}$. This function $\mathcal{P}_{S}$ satisfies $\mathcal{P}_{S}(\phi \circ \psi)=$ $\mathcal{P}_{\psi(S)}(\phi)+\mathcal{P}_{S}(\psi)$ for $\phi, \psi \in \mathcal{E}_{f}$, since

$$
\begin{aligned}
\mathcal{P}_{S}(\phi \circ \psi)= & \frac{1}{2}\left\{\tilde{q}_{f}\left((\phi \circ \psi)_{*}\left(\alpha_{1}+\cdots+\alpha_{2 g+k-1}\right)\right)-\tilde{q}_{f}\left(\alpha_{1}+\cdots+\alpha_{2 g+k-1}\right)\right\} \\
= & \frac{1}{2}\left\{\tilde{q}_{f}\left(\phi_{*}\left(\psi_{*} \alpha_{1}+\cdots+\psi_{*} \alpha_{2 g+k-1}\right)\right)-\tilde{q}_{f}\left(\psi_{*} \alpha_{1}+\cdots+\psi_{*} \alpha_{2 g+k-1}\right)\right\} \\
& +\frac{1}{2}\left\{\tilde{q}_{f}\left(\psi_{*}\left(\alpha_{1}+\cdots+\alpha_{2 g+k-1}\right)\right)-\tilde{q}_{f}\left(\alpha_{1}+\cdots+\alpha_{2 g+k-1}\right)\right\} \\
= & \mathcal{P}_{\psi(S)}(\phi)+\mathcal{P}_{S}(\psi) .
\end{aligned}
$$




\section{REFERENCES}

[1] M. Kato, Topology of bands, Sūrikaisekikenkyūsho Kōkyūroku 243 (1975) 88-96.

[2] T. Nowik, Automorphisms and embeddings of surfaces and quadruple points of regular homotopies, J. Differential Geometry 58 (2001) 421-455.

[3] U. Pinkall, Regular homotopy classes of immersed surfaces, Topology 24 (1985) 421-434.

Department of Mathematics, Faculty of Science and Engineering, Saga UniverSITY, SAGA, 840-8502 JAPAN

E-mail address: hirose@ms.saga-u.ac.jp

Department of Mathematics, Tokyo Gakugei University, Nukuikita 4-1-1, KoGANEI, TOKYO 184-8501 JAPAN

E-mail address: yasuhara@u-gakugei.ac.jp 